\title{
Welcher Erreger hat hier zugeschlagen?
}

— Eine 18-jährige Frau suchte wegen einer ausgedehnten, rötlich livide verfärbten und fluktuierenden Hautveränderung im Bereich der rechten Wange eine Hautklinik auf (Abb. links). Vor sechs Monaten hatte sie sich in einem einschlägigen Etablissement ein Piercing am rechten Tragus anbringen lassen. Etwa vier Wochen später war die strangförmige Hautveränderung über dem rechten Kieferwinkel aufgetreten. Der Hausarzt hatte bereits einen Abszess in dieser Gegend drainiert und mit Cefalexin behandelt, was den Zustand aber nicht verbesserte. Im weiteren Krankheitsverlauf erwog man eine Infektion mit atypischen Erregern und führte eine Biopsie durch. Dabei wurde eine granulomatöse Entzündung festgestellt, es fanden sich aber keine säurefesten Stäb-

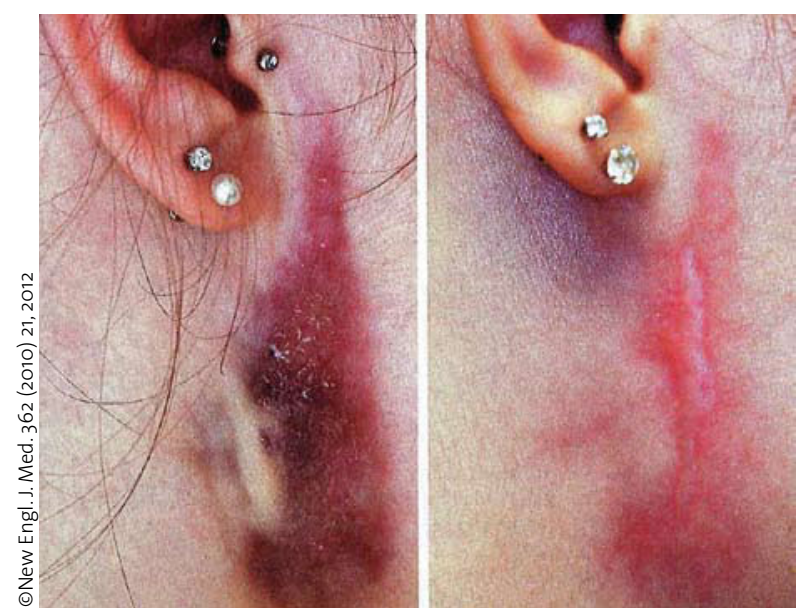

Hautveränderung vor (links) und nach viermonatiger antibiotischer Therapie (rechts). chen. Kulturell konnte Mycobacterium fortuitum nachgewiesen werden. Nach einer vier Monate langen Behandlung mit Ciprofloxacin und Trimethoprim-Sulfamethoxazol trat eine deutliche klinische Besserung ein (Abb. rechts). Nachdem die Patientin wegen eines bekannten Long-QT-Syndroms Betablocker einnahm, vermied man die Verordnung eines Makrolids.

\section{Kommentar}

Viele junge Leute machen sich nicht klar, welche Risiken mit dem Piercing selbst in scheinbar professionell geführten Studios verbunden sind. Für den Kunden derartiger Studios ist es nicht einfach herauszufinden, ob dort einschlägige hygienische Standards eingehalten werden. Abgesehen von den methodenimmanenten Problemen wie Perichondritis, unspezifischen Entzündungen oder Abszedierungen können Infektionen mit Hepatitis B oder C auftreten. Heilt ein entzündlicher Lokalbefund unter Lokaltherapie und systemischer antibiotischer Behandlung nicht $a b$, sollte an die Infektion mit einem atypischen Erreger gedacht werden. Säurefeste Stäbchen sind nicht immer im histologischen Präparat nachweisbar, sodass man eine Isolierung des Erregers in der Gewebekultur versuchen sollte. Nicht tuberkulöse Mykobakterien verursachen meist Pusteln, Plaques und Ulzerationen mit ausgesprochen schlechter Abheilungstendenz.

H. S. FüEßL :

- K. A. Horii, M. A. Jackson

(Korr.: Kimberly A. Horii, MD, Children's Mercy Hospitals and Clinics, Kansas City, MO, e-mail: kathorii@cmh.edu): Piercing-related nontuberculous mycobacterial infection. New Engl. J. Med. 362 (2010) 21, 2012

\section{„Binge-Eating“: Was stoppt die Essanfälle?}

Reicht bei der „Binge-Eating“-Störung (BES) ein Gewichtsreduktionsprogramm (GRP) auch zur Behandlung der Essanfälle aus oder ist eine spezialisierte Psychotherapie nötig?

- An zwei Zentren wurden 205 übergewichtige und adipöse Patienten mit BES randomisiert einem von drei Therapiearmen zugewiesen: GRP, interpersonelle Therapie (IPT), therapeutengestützte Selbsthilfe (GSH). Die Therapiedauer betrug 24 Wochen. Nachun- tersuchungen erfolgten ein und zwei Jahre nach Therapieende. Haupt-Outcome-Kriterium war die Anzahl der Tage mit Essanfällen innerhalb des letzten Monats.

Am Therapieende nach 24 Wochen hatten etwa $60 \%$ der Patienten eine Remission der Essanfälle erreicht, wobei kein Unterschied zwischen den Therapieansätzen bestand. Die Abbrecherrate war bei der IPT mit 7\% signifikant niedriger als beim GRP (28\%) und bei der GSH (30\%). IPT und GSH führ- ten signifikant häufiger zur Remission der Essanfälle als das GRP.

\section{Kommentar}

Es wird deutlich, dass man bei der BES gute Therapieerfolge mit hohen Remissionsraten erzielen kann. Die therapeutengestützte Selbsthilfe stellt eine effiziente Behandlung der BES dar. M. DE ZWAAN =

\section{- G. T. Wilson et al.}

Psychological treatments of bingeeating disorder. Arch Gen Psychiatry 67 (2010) 94-101 